\title{
Proposta de uma solução computacional para deteç̧ão de nódulos pulmonares
}

\author{
Fernando Roberto Pereira ${ }^{1}$, Lucas Ferrari de Oliveira $^{2}$ \\ ${ }^{1}$ Instituto Federal de Educação Ciência e Tecnologia de Santa Catarina \\ Santa Catarina - Brasil \\ ${ }^{2}$ Universidade Federal do Paraná \\ Paraná - Brasil \\ fernando.pereiradifsc.edu.br, lferrarieinf.ufpr.br
}

\begin{abstract}
Cancer is one of the leading causes of death worldwide. Recently lung cancer has caused more than 1 million of deaths. Early detection increases the probability of cure. Attempting to aid diagnosis is presented a computational solution for the detection of pulmonary nodules in thorax CT images. The image database used was Lung Imaging Database Consortium. The proposed solution uses the pulmonary area segmentation, pulmonary nodules segmentation and labeling, extraction of characteristics (texture and shape) and pulmonary nodules classification. The segmentation step misses less than $2 \%$ of nodules and the classification scored $78.44 \%$ sensitivity and $88.59 \%$ accuracy. The results indicate that with some adjustments the technique can be promising.
\end{abstract}

Resumo. $O$ câncer ainda é um dos principais motivos de óbitos em todo o mundo. Só o câncer de pulmão causou mais de 1 milhão de mortes recentemente. A detecção precoce aumenta a probabilidade de cura, tentando auxiliar o diagnóstico é apresentada uma proposta de solução computacional para detecção de nódulos pulmonares em imagens de Tomografia Computadorizada de tórax. A base de dados de imagens utilizada foi Lung Imaging Database Consortium. A solução proposta utiliza a segmentação da área pulmonar, segmentação e rotulação de objetos candidatos a nódulos pulmonares, extração de características empregando descritores de textura e forma e classificação dos objetos candidatos a nódulos pulmonares. Na etapa de segmentação menos de $2 \%$ dos nódulos foram perdidos e a classificação ficou com 78,44\% de sensibilidade e 88,59\% de acurácia média. Os resultados indicam que com alguns ajustes a técnica pode ser promissora.

\section{Introdução}

Segundo a World Health Organization (WHO) o câncer é uma das principais causas de morte em todo o mundo, representando 8,8 milhões de mortes em 2015. Sendo que o câncer de pulmão causou aproximadamente 1,69 milhões de mortes. O termo câncer é genérico para um grande grupo de doenças que podem afetar qualquer parte do corpo. Uma característica que pode definir o câncer é a criação rápida de células anormais, que crescem além dos limites habituais e podem se espalhar para outros órgãos, esse processo é denominado metástase[WHO 2017]. 
Soluções computacionais para Computer-Aided Detection (CAD) estão sendo desenvolvidas com o intuito de automatizar o processo de detecção e medição de nódulos com o objetivo de auxiliar profissionais da área da saúde, geralmente, por meio de análise dos exames de Tomografia Computadorizada (TC). Essas soluções geralmente são utilizadas como segunda opinião em diagnósticos [Awai et al. 2004].

De acordo com [Brown et al. 2014], fatores como detecção errônea de elementos normais (ramos vasculares ou vias aéreas) como nódulos (Falso Positivos - FP), influenciam de modo negativo profissionais da área da saúde em relação a utilização desses sistemas. Outro problema citado pelos autores é a falta de padronização e critério para validar soluções CAD. Com o desenvolvimento de base de dados de imagens como a Lung Imaging Database Consortium (LIDC), torna-se possível realizar validação consistente com base em diagnósticos de especialistas.

A base de dados de imagem LIDC consiste em exames de TC de Tórax com documentação dos diagnósticos das lesões encontradas. A base contém exames de 1.010 pacientes em 1.018 casos, totalizando 244.527 imagens. Cada caso inclui TC de tórax e arquivo que registra as anotações dos diagnósticos de radiologistas experientes em formato eXtensible Markup Language (XML) [Armato et al. 2011].

A TC é um exame complementar de diagnóstico por imagem, que é armazenado em formato Digital Imaging and Communications in Medicine (DICOM) [Beutel et al. 2000]. As imagens possuem dimensões de 512 x 512 pixels. Cada pixel pode assumir valores positivos ou negativos, de acordo com a escala de intensidade de Hounsfield Unit (HU) que mensura a densidade dos tecidos. Tecidos com densidades menores que a água (e.g. gordura), os valores HU são negativos e, tecidos com densidades maiores (e.g. ossos e calcificações), apresentam valores positivos. A escala é calculada em relação a água e o ar [Buzug 2008].

De acordo com [Deep et al. 2016], existem duas principais abordagens para identificação de nódulos em imagens: i) baseada em métodos de classificação; e ii) baseada em correspondência de modelos (template matching). Os métodos baseados em classificação utilizam descritores (textura e forma), para representarem imagens. Os métodos baseados em correspondência de modelos podem ser subdivididos em duas categorias: i) métodos baseados na deformação de objetos; e ii) métodos baseados na anatomia de modelos.

Neste artigo, é apresentado uma proposta de solução computacional para detecção de nódulos pulmonares baseada no método de classificação. Tema que tem despertado interesse em diversos grupos de pesquisa em virtude da sua complexidade e importância na área da saúde.

\section{Revisão da literatura}

\subsection{Levantamento bibliográfico}

O trabalho proposto em [Brown et al. 2014], desenvolve um método automático para detecção de nódulos pulmonares, que emprega técnicas de Thresholding, Euclidean Distance Transformation (EDT), segmentação baseada em Watershed e análise sobre componentes conectados. A partir dos máximos EDT locais de cada nódulo candidato é realizada a análise dos pixels conectados para descobrir os vizinhos em torno dele que satisfaçam a 
restrição estabelecida. Para eliminar os não nódulos as restrições são definidas em relação ao volume e forma. Os autores conseguiram com essa abordagem os respectivos resultados, para nódulos com tamanho $\geq 4 \mathrm{~mm}$, sensibilidade de 79,8\% e de 2,05 FP por exame. Para nódulos de tamanho $\geq 8 \mathrm{~mm}$, sensibilidade de $82,2 \%$ e de 1,01 FP por exame.

Os autores em [Firmino et al. 2016], propuseram uma solução computacional para detecção e diagnóstico de nódulos pulmonares. Para segmentação de nódulos utilizaram Watershed e para extração de características dos nódulos empregaram Histogram of oriented Gradients (HOG). Foi utilizado como classificador o Support Vector Machine (SVM) para eliminar os FPs. Com essas técnicas obtiveram 97\% de precisão e 94,4\% de sensibilidade com 7,04 FP por caso. Os experimentos foram realizados com a base da dados de imagens LIDC, para nódulos com diâmetro entre $3 \mathrm{~mm}$ e $30 \mathrm{~mm}$ de diferentes tipos.

A investigação realizada por [Deep et al. 2016], utiliza Local Binary Patterns (LBP) e correspondência de modelos para recuperação de nódulos em imagens de TC de pulmão. O LBP é utilizado para extrair características do pulmão e dos nódulos, foi escolhido por sua capacidade de codificar especificações minuciosas e apresentar baixo custo computacional. A técnica de correspondência de modelos utilizada foi Sum of absolute differences (SAD). Os autores citam o sucesso da utilização do LBP para imagens de mamografia. Os resultados obtidos com nódulos de tamanho $10 \mathrm{~mm}$ no parênquima pulmonar foram, acurácia de mais de 95\%, especifidade mais de $93 \%$ e sensibilidade mais de $98 \%$. Os experimentos foram realizados com a base da dados de imagens LIDC.

Novas abordagens estão sendo propostas para classificação de nódulos pulmonares, por exemplo, o trabalho em [Ciompi et al. 2017], apresenta um sistema de Deep Learning baseado Convolutional Networks. A abordagem consiste em extrair fragmentos de imagens patch 2D de um nódulo de diferentes visualizações (planos). Os nódulos foram organizados em 6 categorias, sendo elas: solid, calcified, part-solid, non-solid, perifissural e spiculated. O sistema foi treinado (1.352 nódulos) e validado (453 nódulos) com a base de dados de imagem Multicentric Italian Lung Detection (MILD), que contém 943 pacientes. Os testes de classificação foram realizados na base de dados de imagens Danish Lung Cancer Screening Trial (DLCST). Para analisar o desempenho do sistema de classificação proposto foi comparado com o diagnóstico de radiologistas experientes. Foi observado que o desempenho médio dos radiologistas é comparável ao desempenho médio do sistema proposto, com acurácia média de $72,9 \%$ e $69,6 \%$, respectivamente.

\subsection{Fundamentação teórica}

\subsubsection{Descritores de características}

O descritor de características LBP considera que a textura de uma imagem pode ser decomposta em um conjunto de pequenas unidades, denominadas unidades textuais. O LBP de um pixel é calculado com base nos níveis de cinza dos pixels vizinhos, deste modo, produzindo um conjunto de unidades textuais [Topi et al. 2000].

O trabalho [Ojala et al. 2002], apresenta o conceito de uniformidade das unidades textuais obtidas com LBP, para mensurar a uniformidade a quantidade de transições entre zeros e uns são calculadas. Havendo um número de transições $\leq 2$, o padrão é considerado uniforme, leva-se em consideração que o código é tratado como uma lista circular. Deste modo, existem apenas 58 padrões uniformes, os demais padrões, os denominados não 
uniformes são computados juntos, produzindo 59 características.

O descritor HOG tem como concepção que a aparência e forma de um objeto em uma imagem pode ser descrita por meio da distribuição dos gradientes de intensidade dos pixels ou por meio da orientação das bordas. Os passos fundamentais para gerar o descritor pode ser resumido como: cálculo do gradiente em cada pixel, agrupamento dos pixels em células, agrupamento das células em blocos e cálculo do histograma das orientações [Dalal and Triggs 2005].

A transformação Top-Hat é utilizada para extrair pequenos detalhes e objetos de uma imagem, utilizando operação morfológica. Existem dois tipos de transformação TopHat: white e black, dado um elemento estruturante é realizada operação morfologia de abertura ou fechamento, respectivamente. A imagem da transformação Top-Hat é obtida pela subtração da imagem original pela imagem resultante da operação morfologia de abertura ou fechamento [Gonzalez and Woods 2006].

\subsubsection{Aprendizagem de máquina}

Entre as técnicas de aprendizagem de máquina baseada em instâncias, existem aquelas que elaboram suas hipóteses diretamente a partir das próprias instâncias de treinamento [Russell and Norvig 2009]. O algoritmo de classificação de padrões KNN é um dos seus representantes [Cover and Hart 2006]. O conceito chave do aprendizado baseado em instâncias está na propriedade de qualquer ponto de entrada $x$ específico ter probabilidade de ser semelhante às propriedades de pontos na vizinhança de $x$ [Aha et al. 1991].

SVM é um método de aprendizagem que busca encontrar um hiperplano ótimo de separação, que pode ser definido como uma a função de decisão linear com margem máxima entre os vetores de duas classes. Para problemas linearmente separáveis, o SVM determina o hiperplano com maior margem entre as classes. Para problemas que não são linearmente separáveis, faz-se necessário mapear o conjuntos de dados original para um novo espaço de maior dimensão denominado feature space, deste modo, torna-se possível obter um hiperplano que separe as classes [Vapnik 1995].

\section{Metodologia}

\subsection{Organização da base de dados de imagens}

Os experimentos foram realizados com a base da dados de imagens LIDC, nódulos com diâmetro entre $3 \mathrm{~mm}$ e $30 \mathrm{~mm}$ de diferentes tipos. Para organizar as informações da base de dados foi necessário filtrar os dados e simplificar o conjunto de arquivos que a compõem. A base de dados é composta por exames de diferentes tipos de modalidade, sendo eles CT, Computed Radiography (CR) e Digital Radiography (DX). A solução apresentada neste artigo utiliza apenas os exames da modalidade CT, as imagens estão orientadas no eixo axial.

Cada exame possui registro dos diagnósticos de quatro radiologistas experientes realizados em duas fases. Na primeira fase ocorreu a leitura cega, cada radiologista analisou independentemente cada imagem e marcou lesões pertencentes a uma de três categorias (i.e. nódulo $\geq 3 \mathrm{~mm}$, nódulo $<3 \mathrm{~mm}$ e não nódulo $\geq 3 \mathrm{~mm}$ ). $\mathrm{Na}$ segunda fase, não cega, cada radiologista revisou suas próprias marcas e as marcas anônimas dos outros três 
radiologistas para dar uma opinião final. O objetivo deste processo foi identificar de forma mais completa possível todos os nódulos pulmonares em cada CT [Armato et al. 2011].

Foi utilizada a lista de nódulos descritos em [Reeves and Biancardi 2011]. A mesma foi criada para garantir que vários grupos de pesquisa usem o mesmo conjunto de dados de nódulos. A lista possui dados de cada nódulo, assim é possível estabelecer a localização de cada um deles nas imagens, informações imprescindíveis para as etapas de segmentação e rotulação de objetos candidatos a nódulos.

As imagens de CT de tórax apresentam ruídos dos sensores de imageamento, tecidos, cartilagem, ossos, órgãos etc. Foi necessário remover regiões das imagens que não pertencem ao pulmão para simplificar a detecção de candidatos a nódulos, deste modo, reduzindo o número de estruturas presentes nas imagens.

\subsection{Segmentação da área pulmonar}

Para segmentar a área pulmonar foi realizada a operação binary threshold entre a faixa de valores -1000 HU e -200 HU, valores escolhidos com base no trabalho proposto em [Firmino et al. 2016]. De acordo com os autores esses valores abrangem tecidos, vasos pulmonares e o ar dentro dos pulmões.

Mesmo com a restrição da faixa de valores, estruturas externas ao pulmão permaneceram. Processando as imagens no plano anatômico transverso (que divide o corpo em parte superior e inferior) e sabendo que a área pulmonar está na região central das imagens, foi utilizada a estratégia de elencar objetos da imagem que estão dentro do intervalo de raio 15 a 30 pixels da posição central da imagem, a métrica utilizada foi distância Euclidiana. De cada objeto encontrado é obtido sua posição, coordenadas x, y e z, com essas coordenadas foi criado uma lista de sementes. Com base na lista de sementes foi utilizada a operação region growing (com a mesma faixa de valores -1000 e -200). Deste modo, a partir das sementes foi possível agrupar os pixels vizinhos que estão dentro da faixa de valores.

No total foram geradas 4 máscaras da área pulmonar segmentada, a Figura 1 mostra a imagem original e suas quatro máscaras. A Figura 1b) apresenta a máscara com base apenas no contorno dos pulmões; a Figura 1c) apresenta a operação de dilatação de raio 1 utilizando o elemento estruturante sitkBall $^{1}$, sob o contorno dos pulmões (Figura 1b); já a Figura 1d) mostra o resultado da aplicação da técnica de Convex hull sob o contorno dos pulmões (plano Euclidiano bidimensional) e na Figura 1e) é apresentada a operação de dilatação de raio 1 na Figura 1d).

Para mensurar a eficiência das máscaras em relação ao problema foi verificado quantas marcações de nódulos realizadas pelos especialistas ficaram fora da máscara. Foram estabelecidas 3 categorias: i) totalmente dentro da máscara ( $\geq 20 \%$ dos pontos de cada marcação estão dentro da máscara); ii) parcialmente dentro da máscara $(<20 \%$ dos pontos de cada marcação estão dentro da máscara); e iii) fora da máscara (nenhum ponto de cada marcação está dentro da máscara). A máscara obtida com Convex hull sob o contorno dos pulmões obteve melhor resultado em relação ao número de marcações de nódulos dentro da máscara. Os resultados quantitativos comparando os 4 tipos de

\footnotetext{
${ }^{1}$ Elemento estruturante definido na biblioteca Simple Insight Segmentation and Registration Toolkit (SimpleITK).
} 


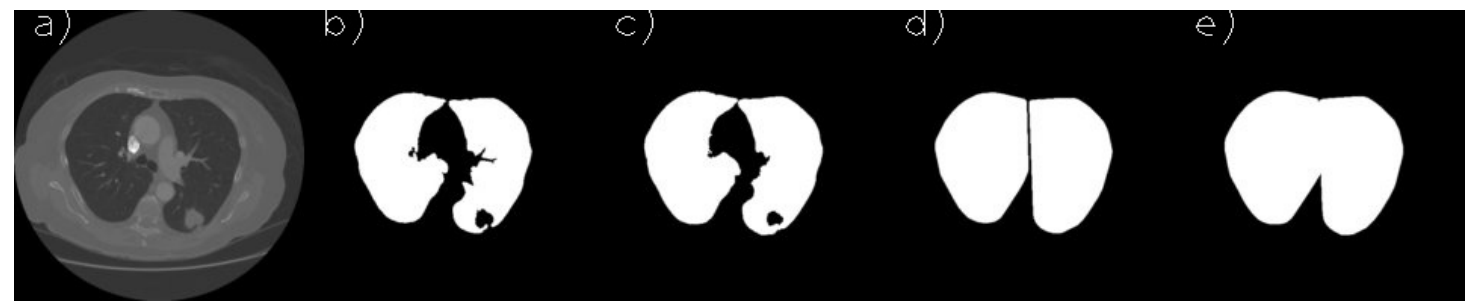

Figura 1. a) Imagem original; b) Contorno dos pulmões; c) Contorno dos pulmões com dilatação de raio 1; d) Convex hull sob o contorno dos pulmões e Convex hull sob o contorno dos pulmões com dilatação de raio 1.

máscaras estão na seção 4.

De modo geral, sistemas de detecção de nódulos pulmonares baseados em classificação utilizam pipeline com duas etapas: i) proposição de objetos candidatos a nódulos. Neste trabalho foi testado processamento digital de imagem por meio das operações threshold e region growing (descrito na seção 3.3); e ii) redução de falsos positivos. Neste trabalho foi testado diferentes descritores de características para representar os objetos candidatos a nódulos, os quais foram classificados, deste modo, reduzindo o número de objetos propostos candidatos a nódulos (descritos nas seções 3.4 e 3.5, respectivamente).

\subsection{Segmentação e rotulação de objetos candidatos a nódulos}

Com a área pulmonar segmentada a próxima etapa foi a segmentação e rotulação de objetos candidatos a nódulos. Foi utilizada a operação de threshold com a faixa de valores $-1000 \mathrm{HU}$ e $-200 \mathrm{HU}$. O resultado obtido foi um conjunto de objetos que possuem coordenadas $\mathrm{x}, \mathrm{y}$ e $\mathrm{z}$ formando uma lista de sementes. A operação region growing utilizou a lista de sementes criada com os valores $-600 \mathrm{HU}$ e $600 \mathrm{HU}$.

Por meio de análise de vários experimentos realizados, foi identificada a perda de alguns objetos candidatos a nódulos, essa perda ocorre principalmente pelo processo de segmentação da área pulmonar, que não preservam as estruturas que estão na extremidade do pulmão. Para amenizar esse problema, antes de realizar a detecção dos objetos candidatos a nódulos, foi utilizado o operador lógico $O R$ com as imagens segmentadas e imagens com o contorno do Convex hull com espessura de 2 pixels. A figura 2 ilustra o resultado obtido com essa operação.

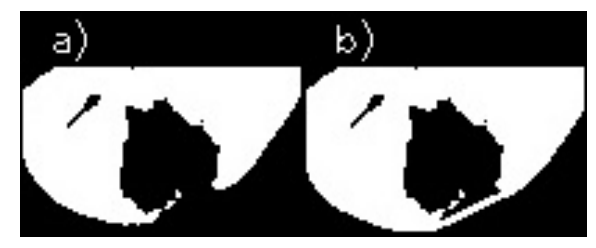

Figura 2. a) Exemplo de objeto que não seria detectado e b) Imagem resultante com o operador lógico $O R$ entre a imagem segmentada e imagem com o contorno do Convex hull.

A etapa de rotulação atribui uma das classes, nódulo ou não nódulo, aos objetos. Para todo objeto encontrado em cada slice foi verificado se pelo menos 1 ponto do seu contorno está dentro de um dos bounding box, caso a condição seja verdadeira, é atribuído o rótulo nódulo ao objeto, caso contrário, é atribuído a classe não nódulo. O bounding 
box é definido com base no conjuntos de todas as marcações realizados pelos especialistas para cada slice onde o nódulo está presente.

\subsection{Extração de características}

Para a extração de características é verificado a qual classe o objeto pertence, sendo da classe nódulo, o descritor utiliza o bounding box estabelecido pelas marcações dos especialistas, caso contrário, pertencente a classe não nódulo, o descritor utiliza o bounding box criado com o contorno do objeto.

Foram testados 3 descritores de característica, sendo eles: i) LBP com variação de padrões uniformes, com os parâmetros raio 1 e com o número de pontos 8 . Com esse descritor foram extraídas 59 características; ii) transformação TOP-HAT em suas abordagens white e black, com o elemento estruturante gerado com skimage.morphology.disk ${ }^{2}$ de raio 3. Com esses descritores foram extraídas 4 características, média e desvio padrão de cada abordagem; e iii) HOG como os respectivos parâmetros número de pixels por célula igual ao tamanho do bounding box, célula por bloco igual a 1 e, com o número de orientações 9 , ou seja, a cada $20^{\circ}$. Com esse descritor foram extraídas 9 características.

\subsection{Experimentos de classificação}

Foram realizados 6 experimentos de classificação com as características extraídas para classificar os objetos segmentados, sendo eles: i) transformação TOP-HAT com suas abordagens white e black; ii) HOG; iii) LBP uniforme; iv) combinando os descritores LBP uniforme mais TOP-HAT com suas abordagens white e black, totalizando 63 características; v) combinando os descritores LBP uniforme mais HOG, totalizando 68 características; e vi) combinando os descritores LBP uniforme mais TOP-HAT com suas abordagens white e black mais HOG, totalizando 72 características.

Para mitigar possíveis problemas com overfitting, foi empregada a abordagem de validação cruzada Leave-One-Patient-Out (LOPO). Outra estratégia utiliza foi balancear a base de treinamento, ou seja, deixar o número de exemplos da classe não nódulo igual ao número de exemplos da classe nódulo, faz-se necessário essa abordagem devido ao número de exemplos da classe não nódulo ser desproporcional ao número de exemplos da classe nódulo. A escolha dos exemplos da classe não nódulo ocorreu aleatoriamente. Os dados extraídos foram normalizados utilizando a abordagem Min-max.

Os classificadores testados foram KNN e SVM, para determinar quais parâmetros de configuração utilizar nos classificadores foram realizados testes com diferentes valores de entrada para cada experimento. Foi utilizado como base de validação apenas 1 exame e, para treinamento os exames restantes (todos os teste utilizaram o mesmo conjunto de dados).

Para o classificador KNN, foram realizados testes variando o número de vizinhos de 1 até 15, de um em um. Para o classificador SVM, foi utilizado o GridSearchCV $V^{3}$ para gerar de forma exaustiva valores como parâmetros de entrada, os parâmetros utilizados foram: kernel (linear e RBF); c (soft margin cost function) com os valores de $2.0^{-8}$ até $2.0^{14}$, variando o expoente de dois em dois; e gamma com os valores de $2.0^{-13}$ até $2.0^{3}$, variando o expoente de dois em dois.

\footnotetext{
${ }^{2}$ Elemento estruturante definido com a biblioteca scikit-image [Stéfan van der Walt et al. 2014].

${ }^{3}$ Função disponível na biblioteca scikit-learn[Buitinck et al. 2013].
} 
A tabela 1 apresenta os melhores parâmetros encontrados para cada experimento, em relação aos classificadores KNN e SVM. Os valores dos parâmetros encontrados foram utilizados para classificar os objetos segmentados. Os resultados quantitativos das classificações estão na seção 4.

Tabela 1. Comparação dos testes para determinar os melhores parâmetros para os classificadores KNN e SVM.

\begin{tabular}{|c|c|c|c|c|c|c|}
\hline \multirow{2}{*}{ Descritor } & \multirow{2}{*}{ Classificador } & \multicolumn{4}{|c|}{ Parâmetros } & \multirow{2}{*}{ Acurácia } \\
\hline & & N. vizinhos & kernel & $c$ & gamma & \\
\hline \multirow{2}{*}{ TOP-HAT } & KNN & 4 & - & - & - & $83.24 \%$ \\
\hline & SVM & - & RBF & 1024.0 & 8.0 & $73.76 \%$ \\
\hline \multirow{2}{*}{ HOG } & KNN & 4 & - & - & - & $85.42 \%$ \\
\hline & SVM & - & $\overline{\mathrm{RBF}}$ & 16.0 & 8.0 & $80.36 \%$ \\
\hline \multirow{2}{*}{ LBP } & KNN & 6 & - & - & - & $92.08 \%$ \\
\hline & SVM & - & RBF & 16384.0 & 8.0 & $90.10 \%$ \\
\hline \multirow{2}{*}{ LBP + TOP-HAT } & KNN & 2 & - & - & - & $89.78 \%$ \\
\hline & SVM & - & $\mathrm{RBF}$ & 16384.0 & 8.0 & $92.75 \%$ \\
\hline \multirow{2}{*}{$\mathrm{LBP}+\mathrm{HOG}$} & KNN & 2 & - & - & - & $90.28 \%$ \\
\hline & SVM & - & RBF & 16384.0 & 0.5 & $86.27 \%$ \\
\hline \multirow{2}{*}{$\mathrm{LBP}+\mathrm{TOP}-\mathrm{HAT}+\mathrm{HOG}$} & KNN & 2 & - & - & - & $91.96 \%$ \\
\hline & SVM & - & RBF & 16384.0 & 0.5 & $91.15 \%$ \\
\hline
\end{tabular}

\section{Resultados}

Conforme mencionado anteriormente foi necessário segmentar a área pulmonar para remover regiões que não são de interesse, deste modo, foram testadas 4 tipos de máscaras para verficiar qual delas tem a menor perda de marcações dos especialistas. A tabela 2 apresenta os resultados obtidos, os teste foram realizados com todas as marcações dos especialista, totalizando 40.430 marcações de nódulos. Com exceção da máscara "contorno dos pulmões"as demais máscaras testadas obtiveram mais de $98 \%$ das marcações categorizadas como dentro. Grande parcela das marcações categorizadas como fora fazem parte dos exames em que não foi possível identificar nenhuma área pulmonar após a segmentação.

Tabela 2. Comparação das máscaras testadas para segmentação da área pulmonar.

\begin{tabular}{cccc}
\hline Tipo de máscara & Dentro & Parcial & Fora \\
\hline Contorno dos pulmões & $97.75 \%$ & $0.52 \%$ & $1.73 \%$ \\
Contorno + dilatação raio 1 & $98.38 \%$ & $0.03 \%$ & $1.59 \%$ \\
Convex hull do contorno & $98.46 \%$ & $0.02 \%$ & $1.52 \%$ \\
Convex hull + dilatação raio 1 & $98.01 \%$ & $0.01 \%$ & $1.99 \%$ \\
\hline
\end{tabular}

Após segmentação da área pulmonar, foi realizada a segmentação e rotulação dos objetos candidatos a nódulos. Foram segmentados 24.302 .073 objetos, dos quais, 14.108 foram rotulados como nódulo, ou seja, pelo menos 1 ponto do contorno do objeto segmentado está dentro do bounding box estabelecido pelas marcações dos especialistas. $\mathrm{O}$ conjunto de dados utilizado possui 15.630 marcações de nódulos (as marcações de diferentes especialistas para o mesmo nódulo no mesmo slice foram agrupadas), deste modo, 
foi obtido 90,26\% de identificação das marcações de nódulos por slice. Em 5 exames não foi possível segmentar nenhum objeto, isso ocorreu, pois os valores utilizados na operação de threshold não foram abrangentes o suficiente.

Em relação aos experimentos de classificação, conforme descritos na subseção 3.5, os descritores que obtiveram os melhores resultados foram o LBP uniforme, combinação do LBP uniforme mais a transformação TOP-HAT e a combinação do LBP uniforme mais transformação TOP-HAT mais HOG. A tabela 3 apresenta dados quantitativos dos experimentos de classificação.

Tabela 3. Comparação dos resultados obtidos com os experimentos de classificação.

\begin{tabular}{rccccccc}
\hline \multirow{2}{*}{ Descritor } & \multirow{2}{*}{ Classificador } & \multicolumn{2}{c}{ Sensibilidade } & \multicolumn{2}{c}{ Especificidade } & \multicolumn{2}{c}{ Acurácia } \\
\cline { 3 - 7 } & & Média & $\sigma$ Relativo & Média & $\sigma$ Relativo & Média & $\sigma$ Relativo \\
\hline \multirow{2}{*}{ TOP-HAT } & KNN & $63.53 \%$ & $53.88 \%$ & $84.60 \%$ & $3,34 \%$ & $84,59 \%$ & $3,34 \%$ \\
\cline { 2 - 7 } & SVM & $73,34 \%$ & $49,81 \%$ & $77,20 \%$ & $4,79 \%$ & $77,21 \%$ & $4,77 \%$ \\
\hline \multirow{2}{*}{ HOG } & KNN & $69,85 \%$ & $49,49 \%$ & $86,40 \%$ & $3,06 \%$ & $86,40 \%$ & $3,05 \%$ \\
\cline { 2 - 8 } & SVM & $77,96 \%$ & $47,20 \%$ & $81,78 \%$ & $4,03 \%$ & $81,80 \%$ & $4,02 \%$ \\
\hline \multirow{2}{*}{ LBP } & KNN & $70,00 \%$ & $51,10 \%$ & $90,26 \%$ & $3,89 \%$ & $90,26 \%$ & $3,89 \%$ \\
\cline { 2 - 8 } & SVM & $70,41 \%$ & $51,08 \%$ & $88,84 \%$ & $3,39 \%$ & $88,84 \%$ & $3,39 \%$ \\
\hline \multirow{2}{*}{ LBP + TOP-HAT } & KNN & $59,29 \%$ & $57,63 \%$ & $90,12 \%$ & $2,46 \%$ & $90,11 \%$ & $2,46 \%$ \\
\cline { 2 - 8 } & SVM & $74,78 \%$ & $48,92 \%$ & $90,46 \%$ & $3,00 \%$ & $90,47 \%$ & $2,99 \%$ \\
\hline \multirow{2}{*}{ LBP + TOP-HAT + HOG } & KNN & $63,30 \%$ & $52,39 \%$ & $89,68 \%$ & $2,52 \%$ & $89,67 \%$ & $2,52 \%$ \\
\hline & SVM & $77,91 \%$ & $47,18 \%$ & $85,46 \%$ & $3,33 \%$ & $85,47 \%$ & $3,32 \%$ \\
\hline & KNN & $64,27 \%$ & $53,54 \%$ & $90,96 \%$ & $2,47 \%$ & $90,96 \%$ & $2,47 \%$ \\
\hline
\end{tabular}

\section{Considerações finais}

Este artigo apresenta como contribuição experimentos com técnicas de processamento de imagem para segmentação da área pulmonar e segmentação de objetos candidatos a nódulos pulmonares. Também é apresentado uma estratégia para estabelecer as extremidades da área pulmonar utilizando Convex hull, para minimizar a perda de objetos candidatos a nódulos em regiões próximas a pleura. Testes com diferentes descritores de textura e forma para representar objetos segmentados candidatos a nódulos e resultados de classificação.

A segmentação da área pulmonar obteve bons resultados, considerando as marcações de nódulos realizadas pelos especialistas que foram categorizadas como dentro da máscara 98.46\%, qual é utilizada para segmentar a área pulmonar. Esta etapa é crucial para a solução proposta, pois afeta o desempenho da etapa segmentação dos objetos candidatos a nódulos pulmonares.

Em relação a segmentação dos objetos candidatos a nódulos pulmonares, foi obtido resultado satisfatório em relação ao número de objetos segmentados que pertencem a classe nódulo, pouco mais de $90 \%$. Várias regiões que existem marcações dos especialistas não foram segmentadas com os parâmetros de thresholding testados.

Considerando a classificação dos objetos candidatos a nódulos pulmonares, os descritores testados obtiveram resultados apenas satisfatórios, por não obterem alta taxa de classificação em relação as métricas utilizadas. O melhor resultado obtido foi $77,91 \%$ de sensibilidade, $85,46 \%$ de especificidade e $85,47 \%$ de acurácia. Ocasionado pela alta taxa de classificações erradas, gerando muitos FP e Falsos Negativos. 
Em relação aos classificadores testados KNN e SVM os resultados de classificação foram semelhantes. Com os dados apresentados fica notório que as duas classes (nódulo e não nódulos) do problema não são linearmente separáveis com os descritores utilizados, em todos os experimentos o SVM encontrou como melhor parâmetro o kernel RBF e precisou utilizar valores altos para soft margin cost function.

Novos experimentos podem ser realizados com a solução proposta, testando novos parâmetros de thresholding nas etapas de segmentação e empregando novos descritores de características para representar os objetos candidatos a nódulos.

Soluções empregando deep learning estão em evidência por apresentar excelentes resultados sem dependerem da intervenção do usuário para definir técnicas de segmentação ou estabelecer descritor de características. Novos experimentos com diferentes arquiteturas e configurações podem ser testadas com o intuito atacar o problema de detecção de nódulos pulmonares.

\section{Referências}

Aha, D., Kibler, D., and Albert, M. (1991). Instance-based learning algorithms. pages 37-66.

Armato, S., McLennan, G., Bidaut, L., McNitt-Gray, M., Meyer, C., Reeves, A., Zhao, B., Aberle, D., Henschke, C., Hoffman, E., Kazerooni, E., MacMahon, H., Beek, E., Yankelevitz, D., Biancardi, A., Bland, P., Brown, M., Engelmann, R., Laderach, G., Max, D., Pais, R., Qing, D., Roberts, R., Smith, A., Starkey, A., Batra, P., Caligiuri, P., Farooqi, A., Gladish, G., Jude, M., Munden, R., Petkovska, I., Quint, L., Schwartz, L., Sundaram, B., Dodd, L., Fenimore, C., Gur, D., Petrick, N., Freymann, J., Kirby, J., Hughes, B., Casteele, A. V., Gupte, S., Sallam, M., Heath, M., Kuhn, M., Dharaiya, E., Burns, R., Fryd, D., Salganicoff, M., Anand, V., Shreter, U., Vastagh, S., Croft, B., and Clarke, L. (2011). The lung image database consortium (LIDC) and image database resource initiative (IDRI): A completed reference database of lung nodules on CT scans. Medical Physics, 38(2):915-931.

Awai, K., Murao, K., Ozawa, A., Komi, M., Hayakawa, H., Hori, S., and Nishimura, Y. (2004). Pulmonary nodules at chest ct: Effect of computer-aided diagnosis on radiologists' detection performance. Radiology, 230(2):347-352.

Beutel, J., Kundel, H., Metter, R., (Firm), K., and of Photo-optical Instrumentation Engineers, S. (2000). Handbook of medical imaging. volume 1, physics and psychophysics.

Brown, M., Lo, P., Goldin, J., Barnoy, E., Kim, G., McNitt-Gray, M., and Aberle, D. (2014). Toward clinically usable cad for lung cancer screening with computed tomography. European Radiology, 24(11):2719-2728.

Buitinck, L., Louppe, G., Blondel, M., Pedregosa, F., Mueller, A., Grisel, O., Niculae, V., Prettenhofer, P., Gramfort, A., Grobler, J., Layton, R., VanderPlas, J., Joly, A., Holt, B., and Varoquaux, G. (2013). API design for machine learning software: experiences from the scikit-learn project. In ECML PKDD Workshop: Languages for Data Mining and Machine Learning, pages 108-122.

Buzug, T. (2008). From photon statistics to modern cone-beam ct. Springer-Verlag Berlin Heidelberg. 
Ciompi, F., Chung, K., Riel, S., Setio, A., Gerke, P., Jacobs, C., Scholten, E., SchaeferProkop, C., Wille, M., Marchianò, A., Pastorino, U., Prokop, M., and Ginneken, B. (2017). Towards automatic pulmonary nodule management in lung cancer screening with deep learning. Scientific Reports, 7(46479).

Cover, T. and Hart, P. (2006). Nearest neighbor pattern classification. IEEE Trans. Inf. Theor., 13(1):21-27.

Dalal, N. and Triggs, B. (2005). Histograms of oriented gradients for human detection. pages 886-893.

Deep, G., Kaur, L., and Gupta, S. (2016). Lung nodule retrieval by integrating local binary pattern with template matching. American Journal of Computer Science and Engineering Survey, 4(2). ACSES (033-047).

Firmino, M., Angelo, G., Morais, H., Dantas, M., and Valentim, R. (2016). Computeraided detection (cade) and diagnosis (cadx) system for lung cancer with likelihood of malignancy. BioMedical Engineering OnLine, 15(1):2.

Gonzalez, R. and Woods, R. (2006). Digital image processing (3rd edition).

Ojala, T., Pietikäinen, M., and Mäenpää, T. (2002). Multiresolution gray-scale and rotation invariant texture classification with local binary patterns. IEEE Trans. Pattern Anal. Mach. Intell., 24(7):971-987.

Reeves, A. and Biancardi, A. (2011). The lung image database consortium (lidc) nodule size report.

Russell, S. J. and Norvig, P. (2009). Artificial intelligence: a modern approach (3rd edition). Prentice Hall.

Stéfan van der Walt, Johannes Schönberger, Juan Nunez-Iglesias, François Boulogne, Joshua Warner, Neil Yager, Emmanuelle Gouillart, Tony Yu, and the scikit-image contributors (2014). scikit-image: image processing in Python. PeerJ, 2:e453.

Topi, M., Timo, O., Matti, P., and Maricor, S. (2000). Robust texture classification by subsets of local binary patterns. Proceedings 15th International Conference on Pattern Recognition. ICPR-2000, 3:935-938 vol.3.

Vapnik, V. (1995). The Nature of Statistical Learning Theory. Springer-Verlag New York, Inc., New York, NY, USA.

WHO (2017). Cancer. http://www.who.int/mediacentre/factsheets/fs297/en/, Acesso em $06 / 12 / 2017$. 